\title{
Diluted magnetic semiconductors formed by ion implantation and pulsed-laser melting
}

\author{
M.A. Scarpulla ${ }^{1,2}$, U. Daud ${ }^{1,2}$, K.M. Yu², O. Monteiro ${ }^{2}$, Z. Liliental-Weber ${ }^{2}$, D. Zakharov², \\ W. Walukiewicz ${ }^{2}$, and O.D. Dubon ${ }^{1,2}$ \\ ${ }^{1}$ University of California at Berkeley, Berkeley, CA 94720, U.S.A. \\ ${ }^{2}$ Lawrence Berkeley National Laboratory, Berkeley, CA 94720, U.S.A.
}

Using ion implantation followed by pulsed-laser melting (PLM), we have synthesized ferromagnetic films of $\mathrm{Ga}_{1-\mathrm{x}} \mathrm{Mn}_{\mathrm{x}} \mathrm{As}$. Ion-channeling experiments reveal that these films are single crystalline and have high $\mathrm{Mn}$ substitutionality while variable temperature resistivity measurements reveal the strong Mn-hole interactions characteristic of carrier-mediated ferromagnetism in homogeneous DMS's. We have observed Curie temperatures ( $\mathrm{T}_{\mathrm{C}}$ 's) of approximately $80 \mathrm{~K}$ for films with substitutional $\mathrm{Mn}$ concentrations of $x=0.04$. The use of n-type counter doping as a means of increasing Mn substitutionality and $\mathrm{T}_{\mathrm{C}}$ is explored by co-implantation of $\mathrm{Mn}$ and $\mathrm{Te}$ into GaAs. In $\mathrm{Ga}_{1-\mathrm{x}} \mathrm{Mn}_{\mathrm{x}} \mathrm{P}$ samples synthesized using our technique, the implanted layer regrows as an epitaxial single crystal capped by a highly defective surface layer. These samples display ferromagnetism with $\mathrm{T}_{\mathrm{C}} \approx 23 \mathrm{~K}$.

Keywords:

GaMnAs, GaMnP, laser processing, ion implantation, diluted magnetic semiconductor

Corresponding Author:

M.A. Scarpulla; scarps@newton.berkeley.edu; Ph: (510) 486-5555; Fax: (510) 486-5530

Dept. of Materials Science \& Engineering, 180 HMMB, UC Berkeley, Berkeley, CA 94720, USA 
Diluted magnetic semiconductors (DMS's), especially ferromagnetic $\mathrm{Ga}_{1-\mathrm{x}} \mathrm{Mn}_{\mathrm{x}} \mathrm{As}$, continue to be intensely investigated for applications in spin-based electronics [1,2]. $\mathrm{Ga}_{1-\mathrm{x}} \mathrm{Mn}_{\mathrm{x}} \mathrm{As}$ exhibits ferromagnetism for $0.02<\mathrm{x}<0.06$ with $\mathrm{T}_{\mathrm{C}}$ 's exceeding $150 \mathrm{~K}$ after post-growth annealing [3-5]. Because the equilibrium solubility of transition metals is orders of magnitude lower than the concentrations required for ferromagnetism in III-V semiconductors, single-phase III-V DMS's are notoriously difficult to produce. Until now, low-temperature molecular beam epitaxy (LT-MBE) has been the only method proven capable of synthesizing epitaxial, single-phase III-V films. Attempts to produce $\mathrm{Ga}_{1-}$ ${ }_{\mathrm{x}} \mathrm{Mn}_{\mathrm{x}}$ As using $\mathrm{Mn}$ ion implantation followed by conventional and rapid thermal annealing (RTA) have invariably led to the formation of (ferromagnetic) secondary phases [6-8]. Previous studies of $\mathrm{Mn}$ in GaP based on LT-MBE and on ion implantation followed by RTA have resulted in polycrystalline films [9-11].

Here we discuss the formation of III-V semiconductor films containing Mn using an alternative, versatile processing route: $\mathrm{Mn}$ ion implantation followed by pulsed-laser melting [12]. The rapid melting and recrystallization associated with PLM kinetically traps implanted species within regrown films at concentrations well in excess of equilibrium solubility limits whilst suppressing the formation of second phases [13].

Semi-insulating GaAs (001) wafers were implanted either with $80 \mathrm{keV} \mathrm{Mn}{ }^{+}$ions or were implanted using the metal vapor vacuum arc (MEVVA) technique which we have described previously [12]. Each implanted sample was irradiated with a single pulse with fluence between $0.3-0.5 \mathrm{~J} / \mathrm{cm}^{2}$ from a $\mathrm{KrF}$ excimer laser $(\lambda=248 \mathrm{~nm}, 38 \mathrm{~ns}$ FWHM) homogenized by a crossed cylindrical lens homogenizer. The structure of the layers and the location of $\mathrm{Mn}$ in the lattice were evaluated by $2 \mathrm{MeV}^{4} \mathrm{He}^{+}$channeling 
Rutherford backscattering spectrometry (c-RBS) and particle induced X-ray emission (PIXE) along [001] and [011] directions [4]. A DC-SQUID magnetometer was used to measure the in-plane magnetic behavior of the films along a $<100>$ direction. Temperature-dependent electrical resistivity was measured in the van der Pauw geometry using pressed In contacts.

Using c-RBS and PIXE, we find that the films are single-crystalline and that a large fraction of $\mathrm{Mn}$ is substitutional [12]. For example, the $<110>$ minimum backscattered yield $\left(\chi_{\min }\right)$ is less than 0.046 for a film implanted with $5.2 \times 10^{15} / \mathrm{cm}^{2} 80$ $\mathrm{keV} \mathrm{Mn}^{+}$and regrown with $0.36 \mathrm{~J} / \mathrm{cm}^{2}$, which approaches the $\chi_{\min }$ of 0.027 for an asreceived wafer. Roughly $65 \%$ of the $\mathrm{Mn}$ exists as $\mathrm{Mn}_{\mathrm{Ga}}$ while the remaining fraction occupies non-commensurate locations, e.g. structural defects or the surface of the film. The $\mathrm{Mn}_{\mathrm{Ga}}$ fraction typically decreases in our films with increasing Mn composition (to $40 \%$ for $\mathrm{x}=0.10$ ); however less than a few percent of the Mn in our films occupies interstitial sites. We attribute this absence of interstitials to annealing while the recrystallized film cools from its solidification temperature. This is in contrast to LTMBE films where post-growth annealing is required to excise compensating interstitials [4].

The main panel of Fig. 1 presents the temperature variation of magnetization in an applied field of 50 Oe for a sample implanted with $\mathrm{Mn}$ to $7.5 \times 10^{15} / \mathrm{cm}^{2}$ using MEVVA and irradiated at $0.3 \mathrm{~J} / \mathrm{cm}^{2}$. The $\mathrm{T}_{\mathrm{C}}$ of $64 \mathrm{~K}$ was determined by extrapolating the steep linear portion of the curve to zero. Inset a) depicts the peak in the temperature variation of sheet resistivity around $\mathrm{T}_{\mathrm{C}}$ that is characteristic of carrier-mediated ferromagnetism in DMS's [14]. The variation of magnetization with magnetic field is presented in inset $b$ ) 
and reveals a fairly square hysteresis loop which is similar to those observed in MBEgrown films [15] and is indicative of good crystal quality. Measurements between 2-5 T reveal that the moment per implanted $\mathrm{Mn}$ is at least $2.3 \mu_{\mathrm{B}}$.

It has been observed in LT-MBE grown $\mathrm{Ga}_{1-\mathrm{x}} \mathrm{Mn}_{\mathrm{x}} \mathrm{As}$ that the addition of Be causes substitutional Mn to move into interstitial sites. Based on these experiments, a model was proposed whereby the formation of compensating Mn interstitials is favored as the Fermi energy reaches a certain level with respect to the valence band, resulting in a saturation of the free-hole concentration around $10^{21} / \mathrm{cm}^{3}[16]$. Interstitial $\mathrm{Mn}$ are believed to be double donors and to couple antiferromagnetically to on-site $\mathrm{Mn}$, hence they counter both the electronic and magnetic effects of substitutional Mn. [17]. Yu et al. have proposed that the $\mathrm{T}_{\mathrm{C}}$ can be increased by counter doping films with saturated hole concentrations. The addition of compensating donors would increase the $\mathrm{Mn}_{\mathrm{Ga}}$ concentration by suppressing the formation of Mn interstitials while maintaining the hole concentration at its saturation level [16].

We have investigated this hypothesis by counter doping with Te, for which substitutional incorporation in GaAs at atomic percent levels and with $>90 \%$ substitutionality has been well demonstrated using ion implantation and PLM $[18,19]$. We note that a previous study found that adding $\mathrm{Sn}$ to $\mathrm{Ga}_{1-\mathrm{x}} \mathrm{Mn}_{\mathrm{x}} \mathrm{As}$ decreased $\mathrm{T}_{\mathrm{C}}$ with increasing amounts of $\mathrm{Sn}[20]$. However, the Mn content in these films was well below the level required to saturate the hole concentration. Thus, counter doping reduced $\mathrm{T}_{\mathrm{C}}$ by reducing the free-hole concentration below the saturation level.

We have prepared a series of films sequentially ion implanted with $2.08 \times 10^{16}$ $/ \mathrm{cm}^{2} 80 \mathrm{keV} \mathrm{Mn}{ }^{+}$and varying doses of $160 \mathrm{keV} \mathrm{Te}^{+}$and subsequently regrown using 
PLM. For convenience in the following discussion, we define $\gamma$ as the ratio of retained Te dose to retained Mn dose. Using the combination of c-RBS and PIXE we have determined that counter doping with Te does in fact increase the substitutionality of Mn. The substitutional $\mathrm{Mn}$ fraction in the film containing only $\mathrm{Mn}(\gamma=0)$ is approximately $40 \%$, and this fraction increases to near $60 \%$ and to over $80 \%$ for $\gamma=0.33$ and 1 , respectively. We note that only half of the implanted Mn dose is retained in the $\gamma=1$ sample due to sputtering.

Temperature dependent resistivity measurements of the Te counter doped films reveal that the $\gamma=0$ sample exhibits metallic conduction and a peak near its $T_{C}$ of $77 \mathrm{~K}$, which is a hallmark of critical scattering associated with hole-mediated ferromagnetism in metallic films [5]. The sample with $\gamma=0.33$ is p-type, is more resistive than the other films, and exhibits hopping conduction below $\sim 25 \mathrm{~K}$. Hall effect measurements revealed a reduced hole concentration, which indicates that the layer has been overcompensated. The sample with $\gamma \approx 1$ is n-type with a mean free-electron concentration of approximately $10^{18} / \mathrm{cm}^{3}$.

Associated with the decrease in hole concentration and crossover to n-type conduction in the $\gamma>0$ samples are dramatic changes in the magnetic behavior, as is seen in Fig. 2. The magnetization of the film containing Mn only has a shape typical of our $\mathrm{Ga}_{1-\mathrm{x}} \mathrm{Mn}_{\mathrm{x}} \mathrm{As}$ films and exhibits a $\mathrm{T}_{\mathrm{C}}$ of $\sim 77 \mathrm{~K}$. The n-type film with $\gamma \approx 1$ exhibits only paramagnetism. The intermediate film with $\gamma=0.33$ exhibits a rich temperature dependence involving a change in inflection and a long tail between 25 and $60 \mathrm{~K}$. Magnetization versus applied field measurements on an identical sample reveal an open 
hysteresis loop at $5 \mathrm{~K}$ and a Langevin-shaped magnetization curve with no hysteresis at $45 \mathrm{~K}$.

We have also investigated the behavior of $\mathrm{Mn}$ in $\mathrm{GaP}$ as it has been suggested that $\mathrm{Ga}_{1-\mathrm{x}} \mathrm{Mn}_{\mathrm{x}} \mathrm{P}$ may have a higher $\mathrm{T}_{\mathrm{C}}$ than $\mathrm{Ga}_{1-\mathrm{x}} \mathrm{Mn}_{\mathrm{x}} \mathrm{As}$. Fig. 3 presents a bright-field TEM cross-sectional image taken in a two-beam condition of a GaP film implanted with Mn to $7.5 \times 10^{15} / \mathrm{cm}^{2}$ using MEVVA and regrown using PLM $\left(0.4 \mathrm{~J} / \mathrm{cm}^{2}\right)$. The presence of strain is indicated by the light and dark contrast. This image reveals that implantation and PLM results in single-crystal regrowth for $\sim 150 \mathrm{~nm}$, but that a highly defective layer of $\sim 25 \mathrm{~nm}$ is formed at the surface. No precipitates are apparent in the single crystalline region (region 2). SIMS measurements demonstrate the presence of $\mathrm{Mn}$ at concentrations up to 1.5 at $\%$ in the single crystalline region and channeling RBS and PIXE measurements indicate that $65 \%$ of this $\mathrm{Mn}$ is substitutional (i.e. $\mathrm{x}$ up to 0.02 ). The main panel of Fig. 4 presents the temperature variation of magnetization in a representative sample showing $\mathrm{T}_{\mathrm{C}} \sim 23 \mathrm{~K}$. The inset of Fig. 4 presents the corresponding hysteresis loop obtained at $5 \mathrm{~K}$. We are currently working to determine the origin of this ferromagnetism.

In conclusion, we have demonstrated the synthesis of ferromagnetic $\mathrm{Ga}_{1-\mathrm{x}} \mathrm{Mn}_{\mathrm{x}} \mathrm{As}$ using ion-implantation followed by pulsed-laser melting. We show that the substitutional incorporation of $\mathrm{Mn}$ can be enhanced in $\mathrm{Ga}_{1-\mathrm{x}} \mathrm{Mn}_{\mathrm{x}} \mathrm{As}$ by counter doping with Te. While the addition of Te at the levels described here led to a decrease in $\mathrm{T}_{\mathrm{C}}$ (which, it should be possible to use implantation and PLM to tune the degree of compensation such that the concentration of substitutional $\mathrm{Mn}$ is increased maintaining a saturated hole concentration. It may also be possible to achieve large hole concentrations in such 
material by spatially separating the compensated Mn containing material from heavily $\mathrm{p}$ doped, e.g. by modulation doping or multilayer structures. Finally, we have investigated the regrowth of $\mathrm{Mn}$ ion implanted $\mathrm{GaP}$ and have found that single-crystalline regrowth with high Mn substitutionality is possible using our method.

This work was supported by the Director, Office of Science, Office of Basic Energy Sciences, Division of Materials Sciences and Engineering, of the U.S. Department of Energy under Contract No. DE-AC03-76SF00098. MAS acknowledges support from an NSF Graduate Research Fellowship. ODD acknowledges support from the Hellman Family Fund. 


\section{References}

[1] H. Ohno, J. Cryst. Growth 251 (2003) 285.

[2] T. Dietl, arXiv: cond-mat/0306479 18 Jun 2003.

[3] K.C. Ku et al., Appl. Phys. Lett. 82 (2003) 2302.

[4] K.M. Yu et al., Phys. Rev. B 65 (2002) 201303.

[5] T. Wojtowicz et al., J. Supercond. 16 (2003) 41.

[6] J. Shi et al., Nature 377 (1995) 707.

[7] P.J. Wellmann et al., Appl. Phys. Lett. 71 (1997) 2532.

[8] J. Shi et al., J. Appl. Phys. 79 (1996) 5296.

[9] N. Theodoropoulou et al., Phys. Rev. Lett. 89 (2002) 107203.

[10] M.E. Overberg et al., J. Vac. Sci. Technol., B 20 (2002) 969.

[11] M.E. Overberg et al., Appl. Phys. Lett. 79 (2001) 3128.

[12] M.A. Scarpulla et al., Appl. Phys. Lett. 82 (2003) 1251.

[13] M. Von Allmen and A. Blatter, Laser-beam interactions with materials : physical principles and applications. 2nd ed. Springer, New York, 1995, pp. 89-93.

[14] T. Wojtowicz et al., submitted to Appl. Phys. Lett.

[15] H. Ohno et al., Appl. Phys. Lett. 69 (1996) 363.

[16] K.M. Yu et al., Phys. Rev. B 68 (2003) 041308(R).

[17] J. Blinowski and P. Kacman, Phys. Rev. B 67 (2003) 121204(R).

[18] P.A. Barnes et al., Appl. Phys. Lett. 33 (1978) 965.

[19] J.M. Poate and J.W. Mayer, Laser annealing of semiconductors. Academic Press, New York, 1982.

[20] Y. Satoh et al., Physica E, 10 (2001) 196. 


\section{Figure Captions}

Fig. 1 - (Main] Temperature variation of magnetization with an applied field of 50 Oe for a $\mathrm{Ga}_{1-\mathrm{x}} \mathrm{Mn}_{\mathrm{x}} \mathrm{As}$ sample produced using our technique. The $\mathrm{T}_{\mathrm{C}}$ of $64 \mathrm{~K}$ is indicated by the arrow. (Inset a) Temperature variation of sheet resistivity for an identical sample. The arrow points out the peak near $\mathrm{T}_{\mathrm{C}}$ that is a hallmark of carrier-mediated ferromagnetism. (Inset b) Magnetization vs. applied field at 5 $\mathrm{K}$ for the sample depicted in the main panel.

Fig. 2 - Temperature variation of the magnetization for the samples the Te counter doped $\mathrm{Ga}_{1-\mathrm{x}} \mathrm{Mn}_{\mathrm{x}} \mathrm{As}$ samples.

Fig. 3 - Cross-sectional bright field TEM image of a $\mathrm{Mn}$ ion implanted and PLM regrown GaP film taken in a two-beam condition $(\mathrm{g}=200)$. The damaged surface layer is designated as region 1 , the regrown film is designated as region 2 , while the $\mathrm{GaP}$ substrate is designated as region 3. The contrast in this image is indicative of the presence of strain and dislocations.

Fig. 4 - (main) Temperature variation of magnetization for a $\mathrm{Ga}_{1-\mathrm{x}} \mathrm{Mn}_{\mathrm{x}} \mathrm{P}$ sample. The arrow indicates the $\mathrm{T}_{\mathrm{C}}$ of $23 \mathrm{~K}$. (Inset) Magnetization vs. applied field at $5 \mathrm{~K}$ for the sample depicted in the main panel. 


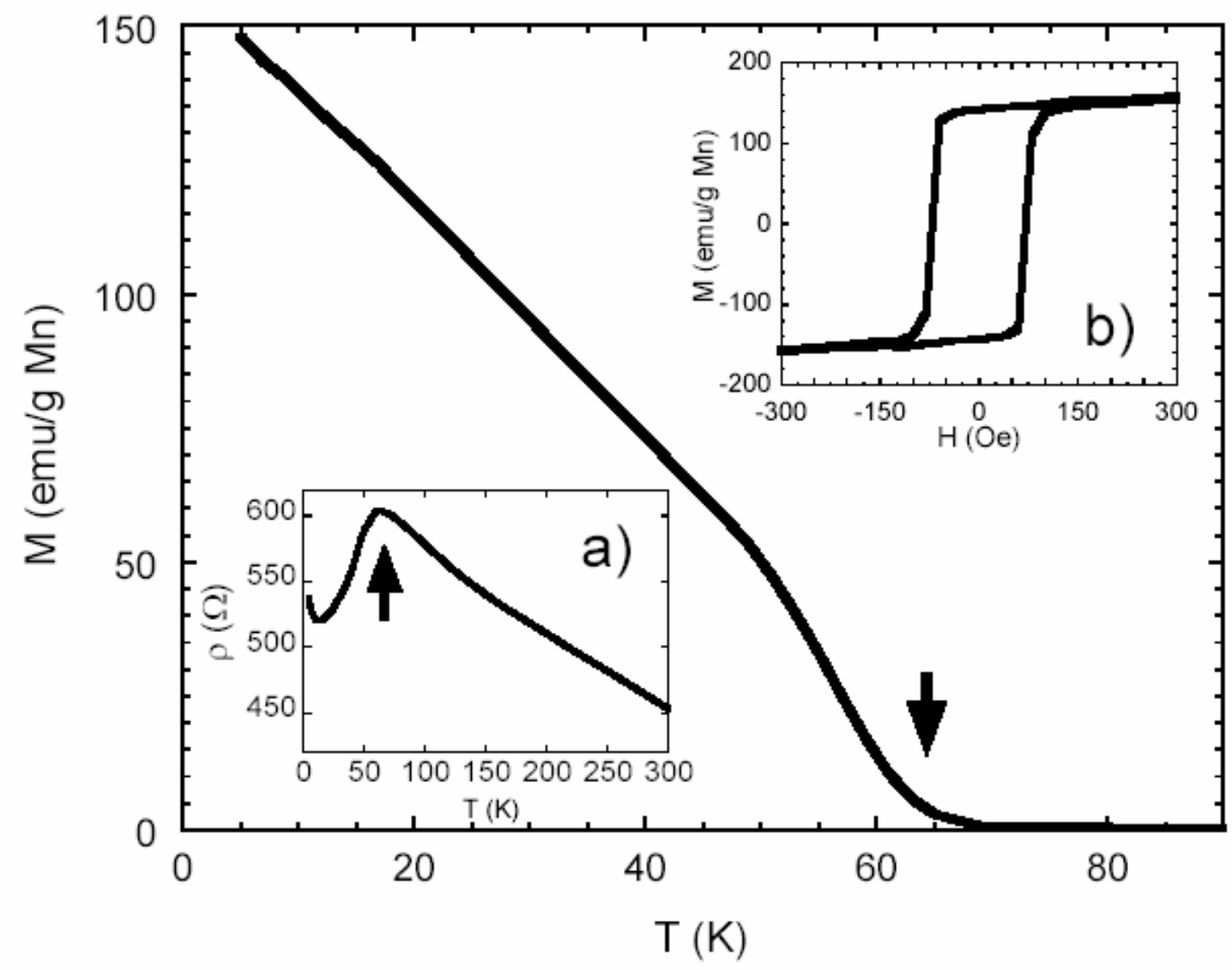

Figure 1 - M.A. Scarpulla et al. 


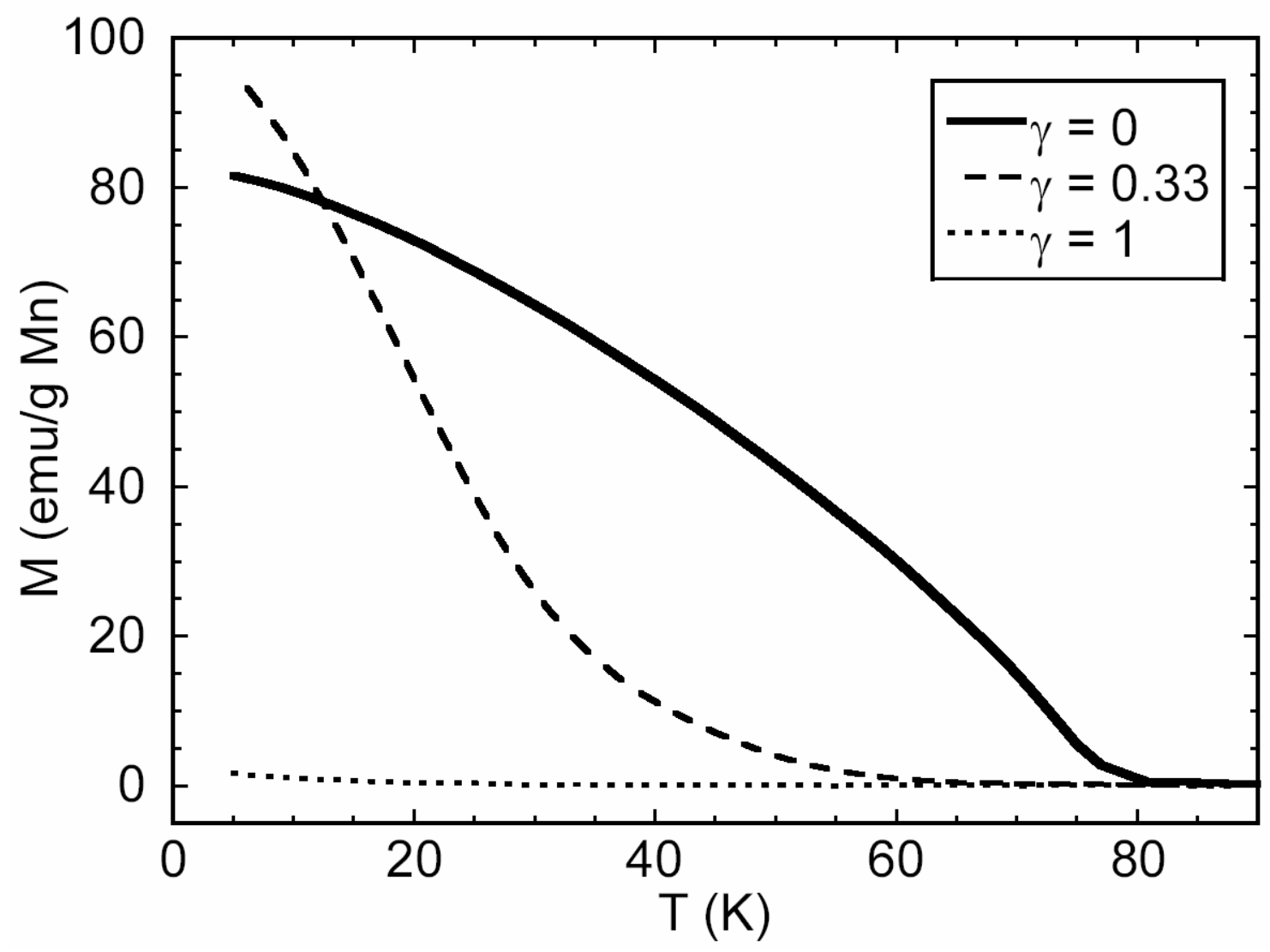

Figure 2 - M.A. Scarpulla et al. 


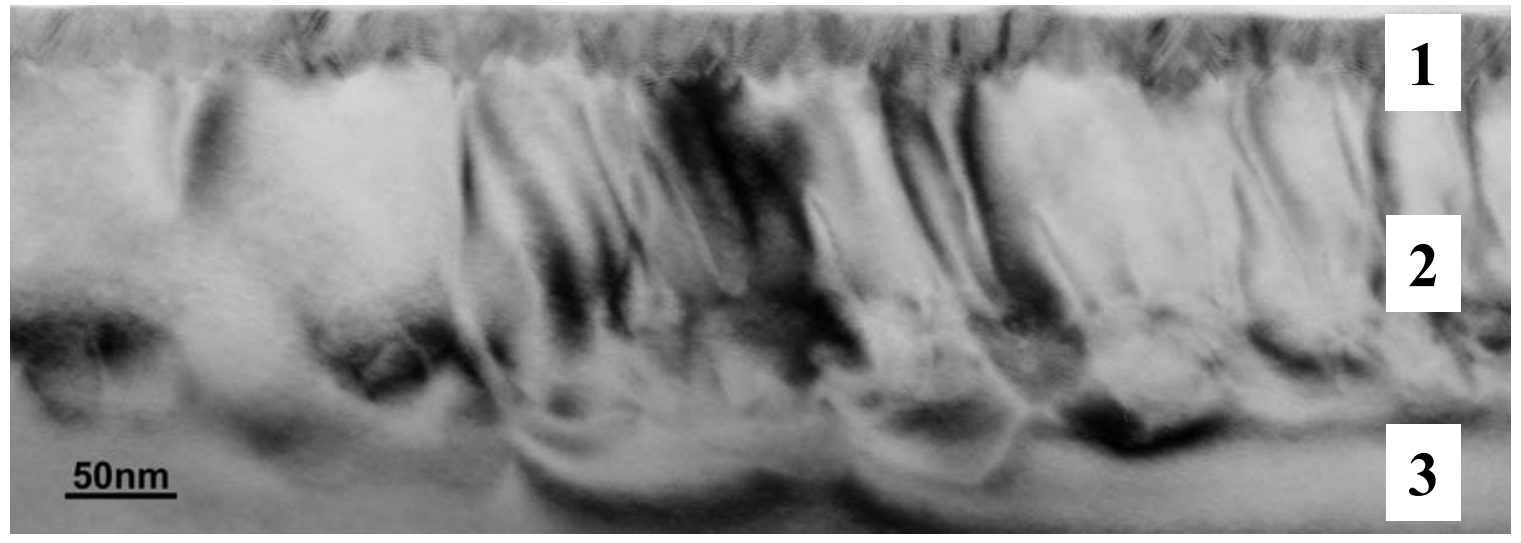

Figure 3 - M.A. Scarpulla et al. 


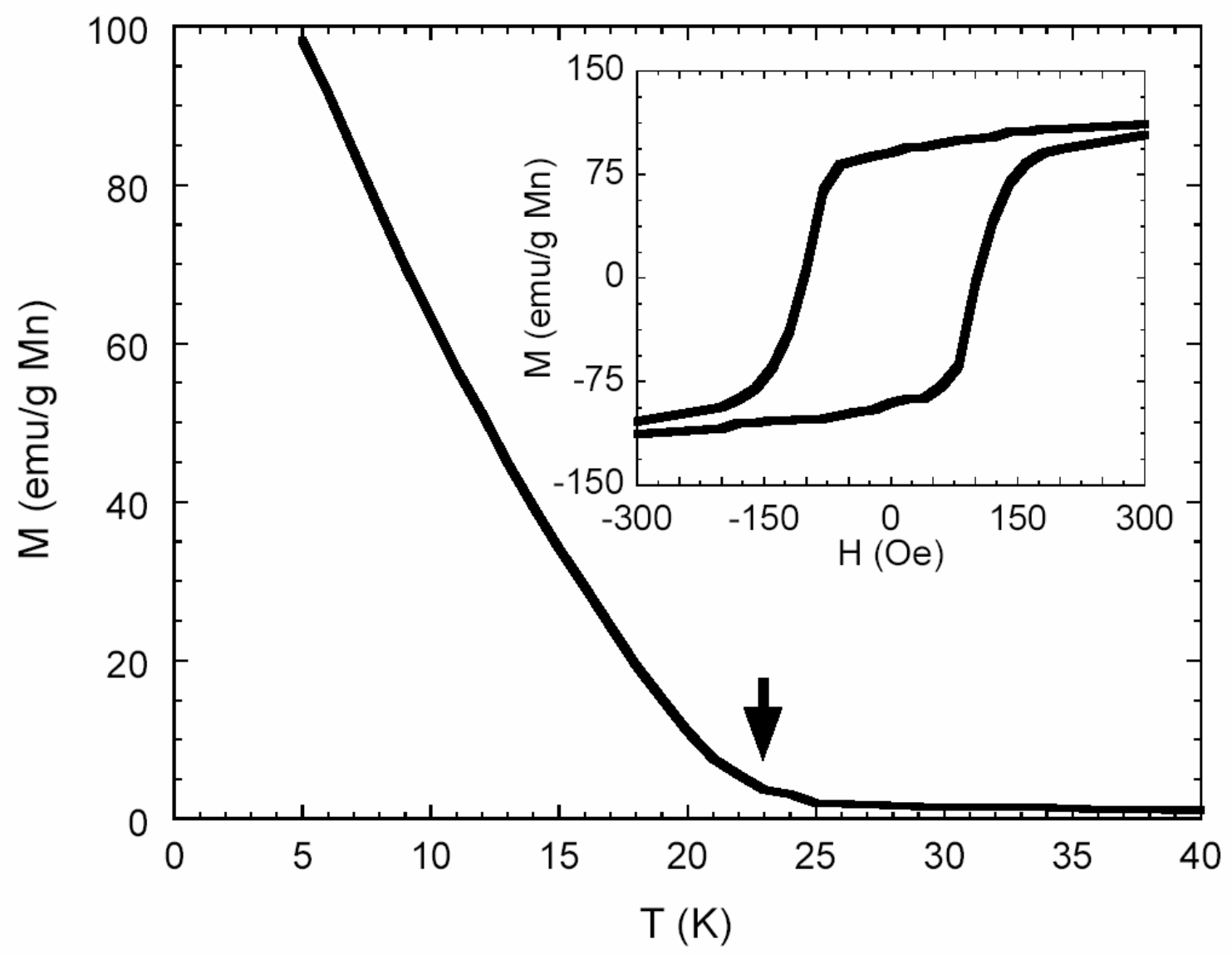

Figure 4 - M.A. Scarpulla et al. 